\title{
Evaluation of HSG and SIS Diagnostic Value in Comparison to Hysteroscopy to Detect Intrauterine Abnormalities in Infertile Women in Iran
}

\author{
Leila Nazari ${ }^{1,2}$ and Parisa Taherzadeh Boroujeni ${ }^{1,2 *}$ \\ ${ }^{1}$ Preventative Gynecology Research Center (PGRC), Shahid Beheshty University of Medical Sciences, Iran \\ ${ }^{2}$ Professor Obstetrician Gynecologist Infertility, IVF center Taleghani Hospital, Iran
}

*Corresponding author: Parisa Taherzadeh Boroujeni, Obstetrician Gynecologist Infertility Fellow, IVF Center, Taleghani Hospital, Iran.

To Cite This Article: Leila Nazari, Parisa Taherzadeh Boroujeni. Evaluation of HSG and SIS Diagnostic Value in Comparison to Hysteroscopy to Detect Intrauterine Abnormalities in Infertile Women in Iran. Am J Biomed Sci \& Res. 2021 - 13(6). AJBSR.MS.ID.001929. DOI: 10.34297/AJBSR.2021.13.001929.

Received: 眥 May 26, 2021; Published: 畊 August 13, 2021

\begin{abstract}
Objective: Hysteroscopy is the gold standard in evaluation of uterine cavity. However, being invasive and its possible adverse effects have reduced popularity of hysteroscopy and clinicians have always been looking for a valid alternative for hysteroscopy. In the current study, we aimed to compare diagnostic value of Saline induced Sonography with HSG in diagnosis of intrauterine abnormalities.

Materials and Methods: We performed a prospective study on 81 infertile women who underwent hysteroscopy, SIS, and HSG at Taleghani infertility center during their IVF treatment course. Polyp, fibroids, adhesion, and septate uterus were considered as abnormality. We reported agreement percent, Kappa, sensitivity, specificity, Area Under Curve, Diagnostic Odds Ratio, Positive Predictive Value, and Negative Predictive value for both SIS and HSG.

Results: Total agreement between SIS and hysteroscopy was 85.1, while it was 23.4 for HSG. We also observed considerably higher Kappa for SIS (80.1\%) than HSG (15.5\%). Overall sensitivity and specificity of SIS for diagnosis of all type of anomaly including both uterine abnormalities and acquired uterine pathologies was $90.1 \%(95 \% \mathrm{CI}=80.7,95.9)$ and $90.0 \%(95 \% \mathrm{CI}=55.5,99.7)$. Meanwhile overall sensitivity and specificity of Hysterosalpingography where hysteroscopy was considered as the gold standard was $54.9(42.7,66.8)$ and $50.0(18.7,81.3)$.
\end{abstract}

Conclusion: SIS is more sensitive tools than HSG for diagnosis of intrauterine abnormalities and could be considered one of the best alternatives of hysteroscopy. It provides more accurate detailed diagnostic information with high sensitivity and specificity.

Keywords: Hysterosalpingography, Sonography, Imaging in Infertility, Intrauterine Abnormalities

\section{Introduction}

Uterine abnormalities are considered as one of the main risk factors in etiology of infertility [1]. According to statistics, the prevalence of uterine abnormalities is high, and it is estimated between $34-62 \%$ and this high prevalence has turned it to one the most common causes of all abnormality cases [2], hence it is estimated between $10-15 \%$ of all infertility cases are due to uterine abnormalities [3]. Therefore, evaluation of uterine cavity is one of the earliest examinations that is regularly performed for infertile women and could considerably increase infertility treatment success rate [4].
Several different diagnostic approaches including Hysterosalpingography (HSG), and hysteroscopy have been established and applied widely over the past years in this regard $[5,6]$. They have been used effectively for many years to investigate uterine cavity abnormalities and provided reliable results. HSG is a low-price, simple, and effective method with high sensitivity in diagnosis of uterine deformities and abnormalities and hysteroscopy has been regarded as the main gold standard in this regard [7]. However, both of hysteroscopy and HSG contains invasive procedure that might be painful and inconvenient for patients $[8,9]$. 
Hysterosonography or Saline Infusion Sonogram (SIS) is one the recently introduced approaches for diagnosis of uterine abnormalities. SIS is an ultrasonic-based diagnostic tool that seems SIS could lead lower adverse effect in comparison to hysteroscopy and HSG due to its non-invasive nature $[10,11]$. Validity of SIS in diagnosis of uterine abnormalities has already been proven, however, in the fast-growing world ultrasonic imaging facilities are developing drastically and results of previous studies implied that the accuracy of such tools have increased over the years. All available attempts reported promising findings for SIS. However, it seems update studies are required [12]. According to one previous study, sensitivity, and specificity of SIS in diagnosis of uterine abnormalities is higher than $80 \%$ [13] while in the earlier studies the reported values were considerably lower [4]. In the current study, we aimed to compare sensitivity and specificity of SIS and HSG in diagnosis of uterine abnormalities in infertile women referred to infertility treatment clinic in Tehran, Iran.

\section{Material and Methods}

\section{Study Participants}

We performed a retrospective study on 81 women aged 20-40 who underwent hysteroscopy, 3-Dimesional Hysterosonography, and HSG at Taleghani infertility treatment center in 2017 in Iran. Hysteroscopy was considered as the gold standard and performed as routine evaluation in treatment of infertility for all study participants. We also performed SIS and HSG for all the eligible cases and compared the results of SIS and HSG with the findings from hysteroscopy. We excluded patients suspected of pregnancy. We also excluded patients with history of severe sensitivity to contrast or iodine, Pelvic Inflammatory Diseases (PID), and acute cervicitis. We trained a radiologist to interpret SIS findings. Moreover, an experienced gynecologist was responsible for performing and interpretation of hysteroscopy and HSG. All procedures were performed between days 7 and 10 of cycle. We used povidoneiodine before the start of SIS procedure to clean cervix and prepare it to enter sonohysterography catheter to the cervical ostium.

We also applied a B-mode endovaginal probe and a $20 \mathrm{~mL}$ syringe that was used for saline solution injection into the uterine cavity. Investigation of intrauterine structure was performed in Uterine abnormalities was the main outcome of the current study and all kinds of polyps, fibroids, adhesion, septate uterus, myomas uterus, bicornuate uterus, hyperplasia, and arcuate uterus were considered as abnormality and those patients without all the mentioned abnormalities were defined as normal cases. Then, we categorized patients into three groups including normal group, uterine abnormalities (arcuate, septate uterus, and bicornuate uterus), and acquired uterine pathologies (hyperplasia, polyp, myomas and adhesion) uterine status in hysteroscopy. We used Parson and Lense [14] classification to differentiate endometrial polyps and fibroid classification was performed based on international classification of Submucous fibroid [15].

\section{Ethics Approval}

The current study was reviewed and approved by ethics and review board of Shahid Behesthti University of Medical Sciences. (Ethics approval)

\section{Statistical Analysis}

We used descriptive analysis to report frequency and proportion of different diagnosed abnormalities in hysteroscopy, SIS, and HSG. We also reported sensitivity, specificity, Positive Predictive Value (PPV), Negative Predictive Value (NPV), Area Under Curve (AUC), and Diagnostic Odds Ratio (DOR) and the associated 95\% CI for SIS and HSG as measures of diagnostic test validities. We also reported agreement percent and Kappa to show whether the findings of SIS and HSG were consistent with hysteroscopy. All statistical analysis was performed using Stata ver 14.1, College Station, Texas, USA. The statistically significant level was considered at P-value $<0.05$.

\section{Results}

We performed the study on 81 infertile women who were referred to Taleghani infertility treatment center in 2018-2020 in Iran. Prevalence of uterus abnormalities was $87.6 \%$ based on hysteroscopy findings. However, it was $80.2 \%$ and $54.3 \%$ in SIS and HSG, respectively. Endometrial polyp was the most prevalent type of abnormalities that was observed in $44.4 \%$ of the study participants followed by Septated uterus (17.2\%) and adhesion (8.6\%) (Table 1). Agreement percent for hysteroscopy and SIS was 85.1, while it was only $23.4 \%$ for HSG. We also estimated Kappa as a measure of agreement between the compared approaches with the gold standard and it was $80.6 \%(95 \% \mathrm{CI}=74.4,85.5)$ and $15.5 \%$ $(95 \% \mathrm{CI}=12.6,18.4)$ for SIS and HSG (Figure 1).

Overall sensitivity and specificity of SIS for diagnosis of all type of anomaly including both uterine abnormalities and acquired uterine pathologies was $90.1 \%(95 \% \mathrm{CI}=80.7,95.9)$ and $90.0 \%$ (95\% CI $=55.5,99.7)$. Meanwhile overall sensitivity and specificity of HSG where hysteroscopy was considered as the gold standard was $54.9(42.7,66.8)$ and $50.0(18.7,81.3)$. 


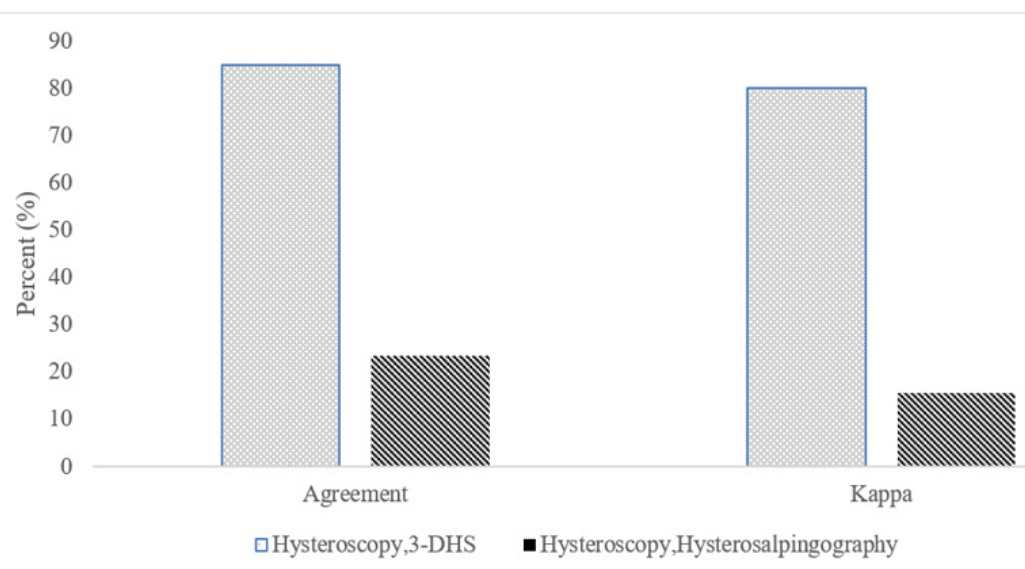

Figure 1: Percentage of agreement between hysteroscopy, SIS, and hysteroscopy, HSG.

Table 1: Frequency of uterine abnormalities and acquired uterine pathologies in Hysteroscopy, SIS, and HSG.

\begin{tabular}{|c|c|c|c|}
\hline Abnormality & Hysteroscopy & SIS & HSG \\
\hline Normal & $10(12.3 \%)$ & $16(19.7 \%)$ & $2(45.6 \%)$ \\
\hline Endometrial Polyp & $36(44.4 \%)$ & $34(41.9 \%)$ & $11(13.5 \%)$ \\
\hline Septate uterus & $14(17.2 \%)$ & $15(18.5 \%)$ & $1(1.2 \%)$ \\
\hline Adhesion & $7(8.6 \%)$ & $7(8.6 \%)$ & $0(0 \%)$ \\
\hline Polyp/ Septate uterus & $4(4.9 \%)$ & $3(3.7 \%)$ & $0(0 \%)$ \\
\hline Polyp/ Uterine Niche & $2(2.4 \%)$ & $2(2.4 \%)$ & $0(0 \%)$ \\
\hline Polyp/ Adhesion & $1(1.2 \%)$ & $1(1.2 \%)$ & $0(\%)$ \\
\hline Hyperplasia & $3(3.7 \%)$ & $0(0 \%)$ & $2(2.4 \%)$ \\
\hline Bicornuate uterus & $1(1.2 \%)$ & $1(1.2 \%)$ & $4(4.9 \%)$ \\
\hline Arcuate uterus & $2(2.4 \%)$ & $0(0 \%)$ & $0(0 \%)$ \\
\hline Myomas uterus & $1(1.2 \%)$ & $1(1.2 \%)$ & $24(29.6 \%)$ \\
\hline Filling detected & $0(0 \%)$ & $1(1.2 \%)$ & $44(54.3 \%)$ \\
\hline All type abnormalities & $71(87.6 \%)$ & $65(80.2 \%)$ & \\
\hline
\end{tabular}

Table 2: Sensitivity and specificity of SIS and HSG versus hysteroscopy in diagnosis of uterine abnormalities in overall and by type of abnormalities.

\begin{tabular}{|c|c|c|c|c|c|c|}
\hline \multirow[b]{2}{*}{ Indexes } & \multicolumn{2}{|c|}{ SIS } & \multirow[b]{2}{*}{ Overall } & \multicolumn{2}{|c|}{ HSG } & \multirow[b]{2}{*}{ Overall } \\
\hline & $\begin{array}{l}\text { Acquired uterine } \\
\text { pathologies }\end{array}$ & $\begin{array}{c}\text { Uterine } \\
\text { abnormalities }\end{array}$ & & $\begin{array}{l}\text { Acquired uterine } \\
\text { pathologies }\end{array}$ & $\begin{array}{c}\text { Uterine } \\
\text { abnormalities }\end{array}$ & \\
\hline Sensitivity $(95 \% \mathrm{CI})$ & $86.8(74.7,94.5)$ & $83.3(58.6,96.4)$ & $90.1(80.7,95.9)$ & $43.4(29.8,57.7)$ & $77.8(52.4,93.6)$ & $54.9(42.7,66.8)$ \\
\hline Specificity $(95 \% \mathrm{CI})$ & $92.9(76.5,99.1)$ & $96.8(89.0,99.6)$ & $90.0(55.5,99.7)$ & $71.4(51.3,86.8)$ & $63.5(50.4,75.3)$ & $50.0(18.7,81.3)$ \\
\hline PPV (95\% CI) & $93.8(89.0,96.5)$ & $87.5(74.4,94.4)$ & $94.4(89.0,97.2)$ & $73.8(60.0,84.1)$ & $37.5(27.1,49.3)$ & $67.1(52.0,79.3)$ \\
\hline NPV (95\% CI) & $78.4(67.4,86.5)$ & $95.6(93.2,97.2)$ & $83.1(70.3,91.1)$ & $40.5(32.3,49.1)$ & $91.0(83.9,95.2)$ & $37.4(22.9,54.6)$ \\
\hline AUC $(95 \% \mathrm{CI})$ & $0.88(0.80,0.95)$ & $0.90(0.81,0.99)$ & $0.901(0.797,1.0)$ & $0.57(0.46,0.68)$ & $0.70(0.59,0.82)$ & $0.525(0.351,0.698)$ \\
\hline DOR (95\% CI) & $54.8(16.7,179)$ & $153(39.7,586)$ & $82.3(70.3,91.1)$ & $1.9(0.7,5.1)$ & $6.0(1.9,19.2)$ & $1.22(0.322,0.698)$ \\
\hline
\end{tabular}

We categorized arcuate, septate uterus, and bicornuate uterus as uterine abnormalities, while hyperplasia, polyp, myomas and adhesion as acquired uterine pathologies and calculated sensitivity and specificity for both distinctively. The estimated sensitivity and specificity for SIS in terms of acquired uterine pathologies was $86.8(74.7,94.5)$ and $92.9(76.5,99.1)$ and it was $83.3(58.6$, $96.4)$ and $96.8(89.0,99.6)$ for diagnosis of uterine abnormalities.
The estimated sensitivity for HSG in diagnosis of either acquired uterine pathologies (Sensitivity $=43.4,95 \% \mathrm{CI}, 29.8,57.7$ ) or uterine abnormalities (Sensitivity=77.8, 95\% CI 52.4, 93.6) was significantly lower than SIS. We observed the same pattern in specificity of HSG. In Table 2, we also reported AUC, PPV, NPV, and DOR as indexes of diagnostic test validities for both 3-SDH and HSG versus hysteroscopy. The overall reported AUC for SIS 
was 0.90 , while it was only 0.525 for HSG in diagnosis of uterine abnormalities. We also reported strongly higher DOR for SIS in comparison to HSG (82.3 versus 1.22).

\section{Discussion}

Evaluation of uterine cavity and early detection of its abnormalities play an essential role in increasing effectiveness of infertility treatments in female as it has been proven as a major risk factor for pathway of infertility in women [5,6]. Hysteroscopy has been used as gold standard in diagnosis of such anomalies, however, it is invasive procedure that needs technical knowledge and facilities to be performed and might lead further complications and adverse effects [16]. Therefore, a validated non-invasive tool has always been interested and couple of ultrasonic based methods have already been developed in this regard $[4,17]$. The current study aimed to compare diagnostic accuracy of SIS as an ultrasound imaging-based approach with HSG in diagnosis of uterine abnormalities compared with the findings of hysteroscopy.

According to our findings agreement percent of SIS with hysteroscopy results was high and it was far better than the reported value for HSG. According to our findings Kappa statistics for SIS was more than $80.0 \%$, whereas it was less than $20 \%$ for HSG. Our findings implied that the results of SIS were identical with findings from hysteroscopy as the gold standard for diagnosis of uterine anomalies. Such a findings were like the previously reported value by De Karoon et al. who reported 84\% accurate diagnosis for SIS [18]. We observed high sensitivity and specificity for SIS that was supported by several previously performed studies. The reported sensitivity and specificity for SIS for detection of uterine abnormalities in a systematic review was $88 \%$ and $94 \%$ respectively [12]. In the current study, the reported values for both sensitivity and specificity of SIS exceeded $90 \%$ and it was inside of previous reports $[17,19,20]$.

The accuracy of SIS remained high when we divided different abnormalities into two groups including uterine abnormalities and acquired uterine pathologies and repeated the analysis separately and high sensitivity and specificity was reported, as well. Our results were inside of all other previous studies that highlighted SIS as a highly sensitive diagnostic modality in diagnosis intrauterine abnormalities including polyps, submucous myomas, intrauterine adhesion, and other intrauterine anomalies $[9,18]$.

We also observed $23.4 \%$ agreement between results of HSG and the Kappa statistics was even lower and reached to $15.5 \%$. The reported sensitivity and specificity for HSG was less than $55.0 \%$ when it compared with hysteroscopy results, and it was considerably lower than the reported values for SIS. Acholonu et al, [13] has reported the same findings and showed that SIS is more effective tool for detection of intrauterine abnormalities in comparison to HSG [13]. Proportion of accurate diagnosis for HSG reported by Acholonu et al was 50.3\% and was significantly lower than SIS [1]. Such findings confirm our results and implies that SIS is practically better alternate of hysteroscopy for diagnosis of intrauterine abnormalities. In one study, Brown et all compared SIS and HSG in respect of uterine abnormalities and observed no statistically significant difference [4]. Over the recent years ultrasonic imaging tools have been drastically developed and it is possible to take3 dimensional images, while it was not available couple of years ago when Brown et al performed their study. Moreover, Brow et al carried out their research on a limited sample size that might affect the validity of their findings [3]. It also might be due to menopause status of study participants. Several studies reported lower sensitivity and specificity for SIS in postmenopausal women than premenopausal ones [12].

Assessing distribution of diagnosis in SIS and HSG shows that in almost $30 \%$ of cases, HSG was unable to determine the exact type of abnormality. Thus, it seems SIS along with higher accuracy provides more detail diagnosis.

The current study was one the least attempts that compared SIS versus HSG and we tried to apply robust statistical approaches for our comparisons. Additionally, we used hysteroscopy as our gold standard and evaluated the results SIS and HSG based on the finding through hysteroscopy. However, couple of limitation must be considered when our findings are interpreted. We performed the study on a relatively small sample size using a retrospective approach and because of that blinding was not applicable.

\section{Conclusion}

It could be concluded that SIS is more sensitive tools than HSG for diagnosis of intrauterine abnormalities and could be considered one of the best alternatives of hysteroscopy. SIS provides more accurate diagnosis with more detailed information, and it is expected to turn the gold standard for diagnosis of intrauterine defects. Therefore, providing widespread public access to SIS in all IVF units and including it into screening programs might be considered as an effective approach that might lead to increased successful fertility rate in females who are candidates of IVF.

\section{Acknowledgement}

We would like to thank all staffs at X infertility center who helped us in data gathering and provided technical supports.

\section{References}

1. Lindemann HJ, Mohr J (1976) CO hysteroscopy: diagnosis and treatment. American Journal of Obstetrics \& Gynecology 124(2): 129-133.

2. For Women's NCC, Children's Health U (2013) Fertility: assessment and treatment for people with fertility problems. 
3. Wallach EE (1972) The uterine factor in infertility. Fertility and sterility 23(2): 138-158.

4. Brown SE, Coddington CC, Schnorr J, Toner JP, Gibbons W, et al (2000) Evaluation of outpatient hysteroscopy, saline infusion hysterosonography, and hysterosalpingography in infertile women: a prospective, randomized study. Fertility and sterility 74(5): 1029-1034.

5. Pundir J, Toukhy TE (2010) Uterine cavity assessment prior to IVF. Women's health 6(6): 841-848.

6. Bingol B, Gunenc Z, Gedikbasi A, Guner H, Tasdemir S, et al. (2011) Comparison of diagnostic accuracy of saline infusion sonohysterography, transvaginal sonography and hysteroscopy. Journal of Obstetrics and Gynaecology 31(1): 54-58.

7. Zeinalzadeh M, Nazari T, Baleggi M (2002) Comparison of hysterosonography and hysterosalpangiography in the diagnosis of intrauterine abnormalities in infertile women. Journal of Reproduction \& Infertility 3(4): 29-35.

8. Vahdat M, Sariri E, Kashanian M, Najmi Z, Monastery A, et al. (2016) Can combination of hysterosalpingography and ultrasound replace hysteroscopy in diagnosis of uterine malformations in infertile women? Med J Islam Repub Iran 30: 352.

9. Prevedourakis C, Loutradis D, Kalianidis C, Makris N, Aravantinos D (1994) Surgery: Hysterosalpingography and hysteroscopy in female infertility. Human Reproduction 9(12): 2353-2355.

10. Hajishaiha M, Ghasemi Rad M, Karimpour N, Mladkova N, Boromand F (2011) Transvaginal sonographic evaluation at different menstrual cycle phases in diagnosis of uterine lesions. International journal of women's health 3: 353-357.

11. Pujar Y, Sherigar B, Patted S, Desai B, Dhumale H (2010) Comparative evaluation of saline infusion sonohysterography and hysterolaparoscopy for diagnosis of uterine cavity abnormalities and tubal patency in infertility: a one-year cross-sectional study. South Asian Fed Obstet Gynecol 2(2): 133-135
12. Seshadri S, El Toukhy T, Douiri A, Jayaprakasan K, Khalaf Y (2015) Diagnostic accuracy of saline infusion sonography in the evaluation of uterine cavity abnormalities prior to assisted reproductive techniques: a systematic review and meta-analyses. Human reproduction update 21(2): 262-274.

13. Acholonu UC, Silberzweig J, Stein DE, Keltz M (2011) Hysterosalpingography versus sonohysterography for intrauterine abnormalities. JSLS 15(4): 471-474.

14. Parsons AK, Lense JJ (1993) Sonohysterography for endometrial abnormalities: preliminary results. Journal of Clinical Ultrasound 21(2): 87-95.

15. Wamsteker K, DeBlok S (1993) Diagnostic hysteroscopy: technique and documentation. Endoscopic surgery for gynaecologists 263-276.

16. Koskas M, Mergui JL, Yazbeck C, Uzan S, Nizard J (2010) Office hysteroscopy for infertility: a series of 557 consecutive cases. Obstetrics and gynecology international 2010: 168096

17. Sylvestre C, Child TJ, Tulandi T, Tan SL (2003) A prospective study to evaluate the efficacy of two-and three-dimensional sonohysterography in women with intrauterine lesions. Fertility and sterility 79(5): 12221225 .

18. de Kroon CD, Jansen FW, Louwé LA, Dieben SW, van Houwelingen HC, et al. (2003) Technology assessment of saline contrast hysterosonography. American journal of obstetrics and gynecology 188(4): 945-949.

19. El Sherbiny W, Nasr AS (2011) Value of 3-dimensional sonohysterography in infertility work-up. Journal of minimally invasive gynecology 18(1): $54-58$

20. Luciano DE, Exacoustos C, Johns DA, Luciano AA (2011) Can hysterosalpingo-contrast sonography replace hysterosalpingography in confirming tubal blockage after hysteroscopic sterilization and in the evaluation of the uterus and tubes in infertile patients? American journal of obstetrics and gynecology 204(1): 79. 